Rapid Communications

\title{
SERS Measurements of Magnetic Stretching Force-Induced Trans-Gauche Conformational Change
}

\author{
Takeyoshi Goto and Hitoshi WATARAI ${ }^{\dagger}$ \\ Department of Chemistry, Graduate School of Science, Osaka University, 1-1 Machikaneyama, \\ Toyonaka, Osaka 560-0043, Japan
}

\begin{abstract}
The effects of stretching forces on covalently bridged 3-mercaptopropanoic acid molecules between magnetic particles (MPs) and Ag nano-particles (NPs) were studied by surface-enhanced Raman scattering (SERS) spectroscopy. With an exertion of $100 \mathrm{pN}$ per single MP, the intensity ratio of the C-S stretching vibrations for trans-to-gauche conformations was increased from $0.295 \pm 0.008$ to $0.69 \pm 0.09$. From the experimental result, it was concluded that the magnetic forces increased the distance between the MP and the Ag NP surface, and induced a shift of the isomerization equilibrium to the trans conformation. The present approach is a new candidate for a dynamic force spectroscopy of conformational equilibria.
\end{abstract}

(Received December 29, 2009; Accepted January 12, 2010; Published February 10, 2010)

An external force applied to molecules varies the Gibbs free-energy curve of the molecules and results in changes in their kinetics and equilibrium. The range of biologically relevant forces is from $\mathrm{pN}$ (van der Waals interactions) to $\mathrm{nN}$ (covalent interactions) for a single bond. ${ }^{1,2}$ The study of the relationship between a mechanical stretching force and the resultant dynamic and equilibrium processes at a molecular level is quite important to invent a new analytical method, which will open a new field of "mechano-chemistry". ${ }^{3-5}$ The present study demonstrates the effects of magnetic stretching forces on the isomerization equilibrium of 3-mercaptopropanoic acid (3-MPA) bridging a magnetic particle (MP) and an $\mathrm{Ag}$ nano-particle (NP), by means of surface-enhanced Raman scattering (SERS) spectroscopy.

Figure 1 shows a schematic illustration of the present experiment. Ag NPs $(62 \pm 21 \mathrm{~nm}$ in diameter $)$, prepared by the reduction of silver nitrate with sodium citrate, were immobilized on a Matsunami adhesive silane coated glass slide (amino-functionalized, Matsunami Glass Ind., Ltd.), which was used as a SERS substrate. The carboxyl groups of 3-MPA molecules $(36 \mathrm{mM})$ were chemically modified to succinimidyl ester with $N$-hydroxysuccinimide $(50 \mathrm{mM})$ and $N$-ethyl- $N^{\prime}$ (3-dimethylaminopropyl) carbodiimide $(50 \mathrm{mM})$, and formed amide bonds with the amine-functionalized MPs $(1 \mu \mathrm{m}$ in diameter, BioMagPlus, Polysciences, Inc.). The 3-MPA bound MPs were thorougly washed with water to remove free 3-MPA molecules. No SERS band attributable to 3-MPA molecules was observed from the liquid solution, itself, and therefore the contribution of Raman scattering from the free 3-MPA molecules was negligible in a following experiment.

To exert uni-directional streching forces on the bridged 3-MPA molecules, magnetic field gradients were applied to MPs on a sample slide with a magnet set, which was arranged with two Nd-Fe-B magnets $(0.55 \mathrm{~T}, 40 \times 80 \times 10 \mathrm{~mm})$ and iron pole

† To whom correspondence should be addressed.

E-mail: watarai@chem.sci.osaka-u.ac.jp pieces $(5 \times 3 \times 2 \mathrm{~mm})$ (Fig. S1, Supporting Information). Two iron pole pieces were set on the magnets to intensify the magnetic flux density to $1.76 \mathrm{~T}$ and its gradients to $2500 \mathrm{~T} / \mathrm{m}$. The gap between the iron pole pieces was set as $0.51 \mathrm{~mm}$. The strength of the stretching forces at the sample position was determined to be $100 \mathrm{pN}$ per MP from microscopic observations of the magnetophoretic velocities of a MP in glycerol using the Stokes drag equation (Fig. S2, Supporting Information).

SERS spectra of covalently bridged 3-MPA molecules were measured with and without external magnetic field gradients. Figure 2 shows the SERS spectra of the 3-MPA monolayer (20 $\mathrm{mM}$, (a)), the bridged 3-MPA without the force exertion

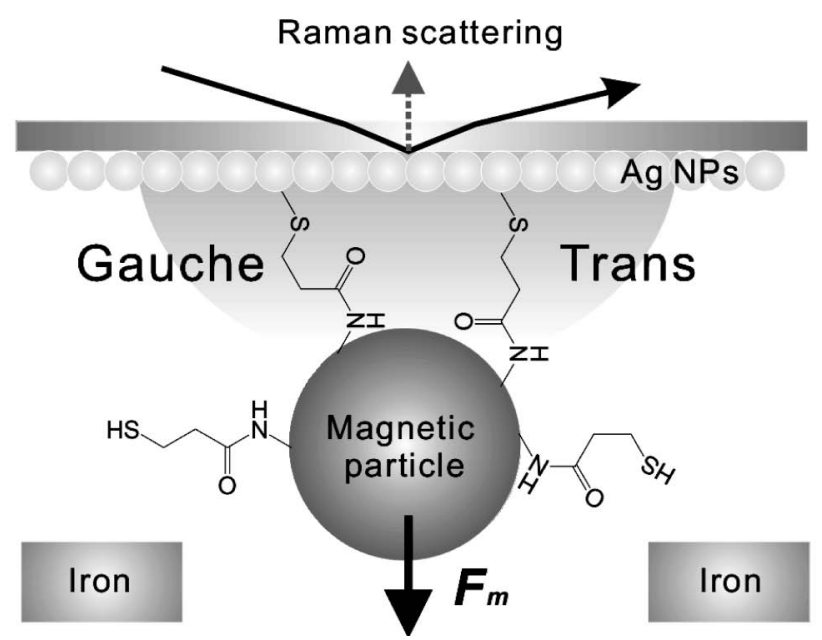

Fig. 1 Schematic illustration of the apparatus (not to scale). 3-Mercaptopropanoic acid molecules are covalently bridging between Ag nano-particles (NPs) on a sample slide and a super-paramagnetic particle. The unidirectional magnetic force $\left(F_{\mathrm{m}}\right)$ was exerted on the magnetic particle by the magnet set. 


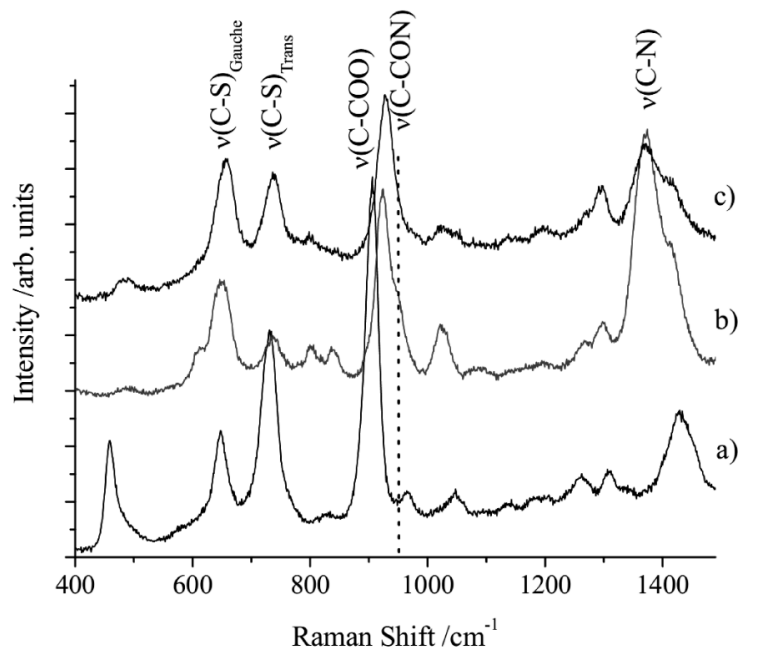

Fig. 2 SERS spectra of 3-mercaptopropanoic acid (3-MPA) monolayer $(20 \mathrm{mM},(\mathrm{a}))$, the bridged 3-MPA without the stretching force $(0 \mathrm{pN}$, (b)), and the bridged 3-MPA with the force exertion $(100 \mathrm{pN},(\mathrm{c}))$. The broken line shows the peak shoulder corresponding to $v(\mathrm{C}-\mathrm{CON})$ at $950 \mathrm{~cm}^{-1}$.

$(0 \mathrm{pN},(\mathrm{b}))$, and the bridged 3-MPA with the force exertion (100 pN, (c)). By comparing Figs. 2(a), (b) and (c), it was found that the Raman frequency of $v(\mathrm{C}-\mathrm{COO})$ was shifted from 906 to $924 \mathrm{~cm}^{-1}$, and $v(\mathrm{C}-\mathrm{CON})$ and $v(\mathrm{C}-\mathrm{N})$ appeared at 950 and $1371 \mathrm{~cm}^{-1}$, respectively, which corresponded to the chemical modification of the carboxyl groups to the amide groups. With the application of a magnetic field gradient, the relative intensity of $v(\mathrm{C}-\mathrm{S})_{\text {trans }} / v(\mathrm{C}-\mathrm{S})_{\text {gauche }}$ was increased as shown in (b) and (c) from $0.295 \pm 0.008(0 \mathrm{pN})$ to $0.69 \pm 0.09(100 \mathrm{pN})$, indicating a shift of the conformational equilibrium to the trans conformation under a forced condition. Also, the intensities of peaks associated with the amide bonds at $950 \mathrm{~cm}^{-1}(v(\mathrm{C}-\mathrm{CON})$, a broken line in Fig. 2) and $1371 \mathrm{~cm}^{-1}(v(\mathrm{C}-\mathrm{N}))$ were decreased for the stretched 3-MPA. Table S1 (Supporting Information) shows the assignments of major vibration bands in the observed SERS spectra. ${ }^{6}$

The number of bridged 3-MPA molecules was estimated from a simple geometric model, which assumed that the perfectly round three Ag NPs $(62 \mathrm{~nm})$, forming a close-packed structure, and the single MP $(1 \mu \mathrm{m})$ were bound through the bridged molecules (the maximum molecular length $=0.76 \mathrm{~nm}$ ). The surface concentration of 3-MPA molecules on a single MP was determined to be $3.29 \pm 0.05 \mathrm{pmole} / \mathrm{cm}^{2}$ from fluorescent emission measurements of 5-(6)-carboxytetramethylrhodamine succinimidyl ester $\left(\lambda_{\mathrm{ex}}=554 \mathrm{~nm}, \lambda_{\mathrm{em}}=585 \mathrm{~nm}\right)$. Then, the maximum number of the briged molecules per single MP was calculated as 8.1 molecules.

Figure 3 shows the optimized forms of the trans and the gauche conformations with the MOPAC program. The length of the trans conformation model for the SC-CC bond is longer than that of the gauche by about $1 \AA$. If the Raman cross-sections of $v(\mathrm{C}-\mathrm{S})_{\text {trans }}$ and $v(\mathrm{C}-\mathrm{S})_{\text {gauche }}$ were assumed to be equal, $\Delta G(100 \mathrm{pN})-\Delta G(0 \mathrm{pN})$ was calculated as $-2.11 \mathrm{~kJ} / \mathrm{mol}$ from the measured intensity ratio $v(\mathrm{C}-\mathrm{S})_{\text {trans }} / v(\mathrm{C}-\mathrm{S})_{\text {gauche. }}$ The applied force per molecule was calculated to be $35 \mathrm{pN}$ from $F_{\mathrm{m}}=$ $(\Delta G(100 \mathrm{pN})-\Delta G(0 \mathrm{pN})) /\left(\Delta x N_{\mathrm{A}}\right)$, where $\Delta x$ is the molecular length difference between the trans and the gauche, which was
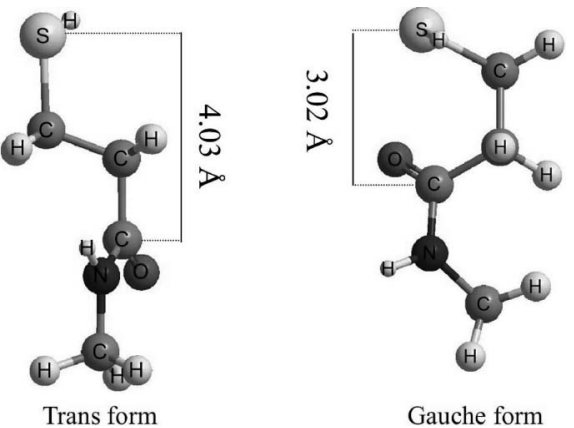

Gauche form

Fig. 3 Optimized forms of bridged molecules with MOPAC of the trans and gauche conformations. The amino group was bound to the magnetic particle (here, methyl amine was used only for modeling). The solid lines show the distance between the $\mathrm{S}$ and $\mathrm{C}$ atoms

estimated as $1 \AA$ from the models, and $N_{\mathrm{A}}$ is the Avogadro constant. Then, the number of bridged 3-MPA molecules was calculated to be 2.9 , which was sufficiently smaller than the result of the fluorescent measurement.

In conclusion, the magnetic stretching force-induced isomerization equilibrium shift from the gauche to trans conformation was observed for the bridged 3-MPA molecules between the MPs and the Ag NPs. The present stretching force/SERS method will be highly promissing for studying of the effect of external forces on the chemical reactions, especially in the mechano-biology relating reactions.

\section{Acknowledgements}

This work was supported by Grant-in-Aid for Scientific Research (S) (No. 16105002) and (A) (No. 21245022) of the Ministry of Education, Culture, Sports, Science and Technology of Japan.

\section{Supporting Information}

A schematic of the force-spectroscopic measurement setup, a graph of the magnetic force of each MP determined from the Stokes drag, and a table of major Raman band assignments. This material is available free of charge on the Web at http://www.jsac.or.jp/analsci/.

\section{References}

1. V. Vogel, Аnпи. Rev. Biophys. Biomol. Struct., 2006, 35, 459.

2. E. Evans, Annu. Rev. Biophys. Biomol. Struct., 2001, 30, 105.

3. J. Zlanatova, S. M. Lindsay, and S. H. Leuba, Prog. Biophys. Mol. Biol., 2000, 74, 37.

4. Y. Iiguni and H. Watarai, Anal. Sci., 2007, 23, 121.

5. C. Danilowicz, D. Greenfield, and M. Prentiss, Anal. Chem., 2005, 77, 3023.

6. J. L. Castro, M. R. Lopez-Ramirez, J. F. Arenas, and J. C. Otero, J. Raman Spectrosc., 2004, 35, 997. 